\title{
Finite-dimensional observer-based PI regulation control of a reaction-diffusion equation
}

\author{
Hugo Lhachemi and Christophe Prieur
}

\begin{abstract}
This paper investigates the output feedback setpoint regulation control of a reaction-diffusion equation by means of boundary control. The considered reaction-diffusion plant may be open-loop unstable. The proposed control strategy consists of the coupling of a finitedimensional observer and a PI controller in order to achieve the boundary setpoint regulation control of various system outputs such as the Dirichlet and Neumann traces. In this context, it is shown that the order of the finite-dimensional observer can always be selected large enough, with explicit criterion, to achieve both the stabilization of the plant and the setpoint regulation of the system output.
\end{abstract}

Index Terms - Reaction-diffusion equation, finite-dimensional observer, output feedback, PI control, boundary regulation control, boundary measurement.

\section{INTRODUCTION}

The problem of controlling the output of a system so as to achieve asymptotic tracking of prescribed trajectories is one of the most fundamental problems in control theory. In the general context of finite-dimensional linear time-invariant (LTI) control systems, the problem of setpoint regulation control is very classical and has been widely investigated. One possible way to solve this problem is based on the augmentation of the state-space representation of the plant with an integral component of the tracking error and the use of the separation principle by exploiting separately a Luenberger observer (which allows the estimation of the state based on the measure only) and a stabilizing full-state feedback (see, e.g., [10]). Even if this approach has reached a very high level of maturity for finitedimensional systems, its possible extension to infinite-dimensional systems, as those considered in this paper, is still an open problem.

Infinite-dimensional systems emerge in many practical applications due to the occurrence of delays, reaction-diffusion dynamics, or even flexible behavior (see, e.g., [12], [16], [17] for introductory textbooks on dedicated control theory for infinite-dimensional systems). While many efficient control design methods have been reported for the stabilization of distributed parameter systems, very few have been extended to the problem of output regulation. The main reason is that all techniques that have been developed for finite-dimensional LTI systems cannot be easily generalized to infinite-dimensional plants. For instance, the frequency domain approach has been generalized to the infinite-dimensional setting, but it requires to deal with an infinite number of poles, yielding an infinite-dimensional pole allocation problem. The state-space approach is followed in this work.

We propose, for the first time, an output feedback control design procedure to achieve the setpoint regulation control of reactiondiffusions system by means of a finite-dimensional observer coupled with a PI controller. The considered reaction-diffusion plant, which might be unstable, is modeled by a Sturm-Liouville operator as those classical introduced in the context of parabolic partial differential

Hugo Lhachemi is with Université Paris-Saclay, CNRS, CentraleSupélec, Laboratoire des signaux et systèmes, 91190, Gif-sur-Yvette, France (e-mail: hugo.lhachemi@centralesupelec.fr). His work has been partially supported by ANR PIA funding: ANR-20-IDEES-0002.

Christophe Prieur is with Université Grenoble Alpes, CNRS, GrenobleINP, GIPSA-lab, F-38000, Grenoble, France (e-mail: christophe.prieur@gipsalab.fr). His work has been partially supported by MIAI@Grenoble Alpes (ANR- 19-P3IA-0003) equation (PDE). The case of PI regulation of this system by means of a state feedback was reported in [14] (see also [3], [4], [8], [14], [15], [20], [24]-[26], [28] for various approaches about PI control design for different types of PDEs). Here we go beyond by designing an output feedback PI control strategy. Even if the proposed procedure also applies to bounded control inputs and bounded observations, we focus the presentation on boundary controls and boundary measurements. This is because these configurations are the most interesting for practical applications and also the most challenging since they involve unbounded control and observation operators (see, e.g., [7] for further explanations). We study several cases for the input-to-output map, covering Dirichlet control inputs (easily extendable to Neumann control inputs as discussed in conclusion) along with Dirichlet and/or Neumann to-be-regulated outputs and measured outputs. We also show that our procedure can be used to regulate a system output that is distinct of the measured one. Therefore, our approach gives a complete framework to study every associated input-to-output maps.

The proposed control design strategy consists of an adequate integral component coupled with a finite-dimensional observer. The design of finite-dimensional observer-based controllers for distributed parameter plants is challenging due to the fact that the separation principle, that is classically used for finite-dimensional systems, does not apply for infinite-dimensional systems [2], [6], [9], [22]. Taking advantage of spectral reduction approaches [5], [21] and using the control architecture initially reported in [22], a LMI-based procedure for solving this stabilization problem for reaction-diffusion PDEs was reported in [11] in the case were the either control or observation operator is bounded. This approach was extended in [13] to the case were both control and observation operators are unbounded, including both Dirichlet and Neumann settings. The present work, taking advantage of [13], goes beyond the simple problem of closedloop stabilization by embracing the issue of output setpoint regulation control. Since the designed observer only estimates a finite number of modes of the infinite-dimensional system, there is an inherent mismatch between the actually measured system output and its estimation as soon as the output is to be regulated to a non-zero value. Hence, one of the main challenges is to account for this mismatch in the dynamics of the observer and then in the subsequent stability analysis. An other challenge is to couple this finite-dimensional observer with a suitable integral component, inspired by the one described in [14] for a state-feedback, in an output feedback setting. Our approach is based on Lyapunov direct methods and the main results take the form of explicit sufficient conditions ensuring the both stability and setpoint regulation control of the closed-loop plant. We assess that these conditions are always feasible provided the order of the observer is selected large enough. Therefore, we show in a constructive manner that the setpoint regulation control of reactiondiffusion PDEs can always be achieved by the coupling of a PI and a finite-dimensional observer.

The paper is organized by considering successively different inputoutput maps for the reaction-diffusion equation depending on the selected boundary measured output, the to-be-regulated output, and the control input. After recalling classical notations and properties for the Sturm-Liouville operators in Section III the case of a Dirichlet 
observation and a Dirichlet control input is considered in Section III Then the case of a Neumann measurement and a Dirichlet control input is considered in Section IV] While the to-be-regulated output and the measured output are the same in the two latter sections, a crossed configuration is considered in Section $\mathrm{V}$ The regulation problem is solved for a Dirichlet measured output, a Neumann tobe-regulated output, and a Dirichlet control input. This final result completes the picture and gives a full study of the different cases for the input-to-output map of the considered class of distributed parameter systems. Some numerical simulations are given in Section VI for this final result. Section VII collects some concluding remarks.

\section{NOTATION AND PROPERTIES}

Spaces $\mathbb{R}^{n}$ are endowed with the Euclidean norm denoted by $\|\cdot\|$. The associated induced norms of matrices are also denoted by $\|\cdot\|$. Given two vectors $X$ and $Y, \operatorname{col}(X, Y)$ denotes the vector $\left[X^{\top}, Y^{\top}\right]^{\top}$. $L^{2}(0,1)$ stands for the space of square integrable functions on $(0,1)$ and is endowed with the inner product $\langle f, g\rangle=\int_{0}^{1} f(x) g(x) \mathrm{d} x$ with associated norm denoted by $\|\cdot\|_{L^{2}}$. For an integer $m \geq 1$, the Sobolev space of order $m$ is denoted by $H^{m}(0,1)$ and is endowed with its usual norm denoted by $\|\cdot\|_{H^{m}}$. For a symmetric matrix $P \in \mathbb{R}^{n \times n}, P \succeq 0$ (resp. $P \succ 0$ ) means that $P$ is positive semi-definite (resp. positive definite).

Let $p \in \mathcal{C}^{1}([0,1])$ and $q \in \mathcal{C}^{0}([0,1])$ with $p>0$ and $q \geq 0$. Let the Sturm-Liouville operator $\mathcal{A}: D(\mathcal{A}) \subset L^{2}(0,1) \rightarrow L^{2}(0,1)$ be defined by $\mathcal{A} f=-\left(p f^{\prime}\right)^{\prime}+q f$ on the domain $D(\mathcal{A}) \subset L^{2}(0,1)$ given by either $D(\mathcal{A})=\left\{f \in H^{2}(0,1): f(0)=f(1)=0\right\}$ or $D(\mathcal{A})=\left\{f \in H^{2}(0,1): f^{\prime}(0)=f(1)=0\right\}$. The eigenvalues $\lambda_{n}, n \geq 1$, of $\mathcal{A}$ are simple, non negative, and form an increasing sequence with $\lambda_{n} \rightarrow+\infty$ as $n \rightarrow+\infty$. Moreover, the associated unit eigenvectors $\phi_{n} \in L^{2}(0,1)$ form a Hilbert basis. We also have $D(\mathcal{A})=\left\{f \in L^{2}(0,1): \sum_{n \geq 1}\left|\lambda_{n}\right|^{2}\left|\left\langle f, \phi_{n}\right\rangle\right|^{2}\right\}$ and $\mathcal{A} f=\sum_{n>1} \lambda_{n}\left\langle f, \phi_{n}\right\rangle \phi_{n}$.

Let $p_{*}, p^{*}, q^{*} \in \mathbb{R}$ be such that $0<p_{*} \leq p(x) \leq p^{*}$ and $0 \leq$ $q(x) \leq q^{*}$ for all $x \in[0,1]$, then it holds [18]:

$$
0 \leq \pi^{2}(n-1)^{2} p_{*} \leq \lambda_{n} \leq \pi^{2} n^{2} p^{*}+q^{*}
$$

for all $n \geq 1$. Assuming further than $p \in \mathcal{C}^{2}([0,1])$, we have for any $x \in\{0,1\}$ that $\phi_{n}(x)=O(1)$ and $\phi_{n}^{\prime}(x)=O\left(\sqrt{\lambda_{n}}\right)$ as $n \rightarrow+\infty$ [18]. Finally, one can check that, for all $f \in D(\mathcal{A})$,

$$
\sum_{n \geq 1} \lambda_{n}\left\langle f, \phi_{n}\right\rangle^{2}=\langle\mathcal{A} f, f\rangle=\int_{0}^{1} p\left(f^{\prime}\right)^{2}+q f^{2} \mathrm{~d} x .
$$

Moreover, for any $f \in D(\mathcal{A})$, we have $f(x)=\sum_{n>1}\left\langle f, \phi_{n}\right\rangle \phi_{n}(x)$ and $f^{\prime}(x)=\sum_{n \geq 1}\left\langle f, \phi_{n}\right\rangle \phi_{n}^{\prime}(x)$ for all $x \in[0,1]$ (see, e.g., [13]).

\section{DiRICHLET MEASUREMENT AND REGULATION CONTROL}

We consider the reaction-diffusion system with Dirichlet boundary observation described for $t>0$ and $x \in(0,1)$ by

$$
\begin{aligned}
z_{t}(t, x) & =\left(p(x) z_{x}(t, x)\right)_{x}+\left(q_{c}-q(x)\right) z(t, x) \\
z_{x}(t, 0) & =0, \quad z(t, 1)=u(t) \\
z(0, x) & =z_{0}(x) \\
y(t) & =z(t, 0)
\end{aligned}
$$

in the case $p \in \mathcal{C}^{2}([0,1])$. Here $q_{c} \in \mathbb{R}$ is a constant, $u(t) \in \mathbb{R}$ is the command input, $y(t) \in \mathbb{R}$ is the measurement, $z_{0} \in L^{2}(0,1)$ is the initial condition, and $z(t, \cdot) \in L^{2}(0,1)$ is the state. The control design objective is to design a finite-dimensional observer-based controller to achieve both stabilization and setpoint regulation control of $y(t)$ to some prescribed reference signal $r(t)$. By setpoint tracking, we mean that our objective is to ensure that $y(t) \rightarrow r_{e}$ when $t \rightarrow+\infty$ as soon as $r(t) \rightarrow r_{e}$ when $t \rightarrow+\infty$ for any arbitrarily given $r_{e} \in \mathbb{R}$.

\section{A. Spectral reduction}

As classically done in the context of boundary control systems (see [7. Sec. 3.3] for details), we start by transforming the nonhomogeneous problem (3) into an equivalent homogeneous one by introducing the change of variable:

$$
w(t, x)=z(t, x)-x^{2} u(t)
$$

that gives the equivalent representation:

$$
\begin{aligned}
& w_{t}(t, x)=\left(p(x) w_{x}(t, x)\right)_{x}+\left(q_{c}-q(x)\right) w(t, x) \\
& +a(x) u(t)+b(x) \dot{u}(t) \\
& w_{x}(t, 0)=0, \quad w(t, 1)=0 \\
& w(0, x)=w_{0}(x) \\
& \tilde{y}(t)=w(t, 0)
\end{aligned}
$$

with $a, b \in L^{2}(0,1)$ defined by $a(x)=2 p(x)+2 x p^{\prime}(x)+\left(q_{c}-\right.$ $q(x)) x^{2}$ and $b(x)=-x^{2}$, respectively, $\tilde{y}(t)=y(t)$, and $w_{0}(x)=$ $z_{0}(x)-x^{2} u(0)$. Introducing the auxiliary command input $v(t)=$ $\dot{u}(t)$, we infer that

$$
\begin{aligned}
\dot{u}(t) & =v(t) \\
\frac{\mathrm{d} w}{\mathrm{~d} t}(t, \cdot) & =-\mathcal{A} w(t, \cdot)+q_{c} w(t, \cdot)+a u(t)+b v(t)
\end{aligned}
$$

with $D(\mathcal{A})=\left\{f \in H^{2}(0,1): f^{\prime}(0)=f(1)=0\right\}$. We introduce the coefficients of projection $w_{n}(t)=\left\langle w(t, \cdot), \phi_{n}\right\rangle, a_{n}=\left\langle a, \phi_{n}\right\rangle$, and $b_{n}=\left\langle b, \phi_{n}\right\rangle$. Considering classical solutions associated with any $z_{0} \in H^{2}(0,1)$ and any $u(0) \in \mathbb{R}$ such that $z_{0}^{\prime}(0)=0$ and $z_{0}(1)=u(0)$ (their existence for the upcoming closed-loop dynamics is an immediate consequence of [19. Chap. 6, Thm. 1.7]), we have $w(t, \cdot) \in D(\mathcal{A})$ for all $t \geq 0$ and we infer that

$$
\begin{aligned}
\dot{u}(t) & =v(t) \\
\dot{w}_{n}(t) & =\left(-\lambda_{n}+q_{c}\right) w_{n}(t)+a_{n} u(t)+b_{n} v(t), \quad n \geq 1 \\
\tilde{y}(t) & =\sum_{i \geq 1} \phi_{i}(0) w_{i}(t)
\end{aligned}
$$

\section{B. Control design}

Let $\delta>0$ be the desired exponential decay rate for the setpoint regulation. We fix $N_{0} \geq 1$ so that $-\lambda_{n}+q_{c}<-\delta<0$ for all $n \geq N_{0}+1$. The integer $N_{0}$, which is definitely fixed for the rest of the control design procedure, can be interpreted as the number of modes that will be "actively" modified by the feedback. We now introduce an arbitrary integer $N \geq N_{0}+1$ which will be further constrained later. Inspired by [22], we design as in [13] an observer to estimate the $N$ first modes of the plant while the state-feedback is performed on the $N_{0}$ first modes of the plant. In this framework, the estimation of the modes ranging from $N_{0}+1$ to $N$ will solely be used to improve the estimate of the system output (see (11)).

Introducing $W^{N_{0}}(t)=\left[\begin{array}{lll}w_{1}(t) & \ldots & w_{N_{0}}(t)\end{array}\right]^{\top}, A_{0}=$ $\operatorname{diag}\left(-\lambda_{1}+q_{c}, \ldots,-\lambda_{N_{0}}+q_{c}\right), B_{0, a}=\left[\begin{array}{lll}a_{1} & \ldots & a_{N_{0}}\end{array}\right]^{\top}$, and $B_{0, b}=\left[\begin{array}{lll}b_{1} & \ldots & b_{N_{0}}\end{array}\right]^{\top}$, we have

$$
\dot{W}^{N_{0}}(t)=A_{0} W^{N_{0}}(t)+B_{0, a} u(t)+B_{0, b} v(t) .
$$

Our objective is to introduce an integral component to achieve the setpoint regulation control of the system output $y(t)$. To do so, we first consider the following classical integral component: $\dot{z}_{i}(t)=$ $y(t)-r(t)=\sum_{n>1} \phi_{n}(0) w_{n}(t)-r(t)$. This $z_{i}$-dynamics, which involves all the modes $w_{n}$ for $n \geq 1$, cannot be embedded into the reduced model (8) that only involves the modes $w_{n}$ for $1 \leq$ $n<N_{0}$. To circumvent this issue, we follow the idea developed in $|14|$ by introducing $\xi_{p}(t)=z_{i}(t)-\sum_{n \geq N_{0}+1} \frac{\phi_{n}(0)}{-\lambda_{n}+q_{c}} w_{n}(t)$ 
whose time derivative is given by $\dot{\xi}_{p}(t)=\sum_{n=1}^{N_{0}} \phi_{n}(0) w_{n}(t)+$ $\alpha_{0} u(t)+\beta_{0} v(t)-r(t)$ with

$$
\alpha_{0}=-\sum_{n \geq N_{0}+1} \frac{a_{n} \phi_{n}(0)}{-\lambda_{n}+q_{c}}, \quad \beta_{0}=-\sum_{n \geq N_{0}+1} \frac{b_{n} \phi_{n}(0)}{-\lambda_{n}+q_{c}} .
$$

The main benefit is that the $\xi_{p}$-dynamics only involves the modes $w_{n}$ for $1 \leq n \leq N_{0}$ while achieving the same equilibrium condition than the $z_{i}$-dynamics. However, in this work and in sharp contrast with the state-feedback setting of [14], the modes $w_{n}$ are not measured. Hence we need to replace them in the dynamics of the integral component by their estimated version $\hat{w}_{n}$ which will be described below. Hence, the employed integral component is described by:

$$
\dot{\xi}(t)=\sum_{n=1}^{N_{0}} \phi_{n}(0) \hat{w}_{n}(t)+\alpha_{0} u(t)+\beta_{0} v(t)-r(t) .
$$

We now define for $1 \leq n \leq N$ the observer dynamics:

$$
\begin{aligned}
\dot{\hat{w}}_{n}(t)= & \left(-\lambda_{n}+q_{c}\right) \hat{w}_{n}(t)+a_{n} u(t)+b_{n} v(t) \\
& -l_{n}\left(\sum_{i=1}^{N} \phi_{i}(0) \hat{w}_{i}(t)-\alpha_{1} u(t)-\tilde{y}(t)\right)
\end{aligned}
$$

with

$$
\alpha_{1}=\sum_{n \geq N+1} \frac{a_{n} \phi_{n}(0)}{-\lambda_{n}+q_{c}}
$$

and where $l_{n} \in \mathbb{R}$ are the observer gains. We set $l_{n}=0$ for $N_{0}+1 \leq$ $n \leq N$. Compared to the stabilization problem studied in [13], we introduce the additional term $-\alpha_{1} u(t)$ in the observer dynamics 11 . This term is added to compensate the inherent steady-state mismatch between the actually measured system output $\tilde{y}(t)$ and its estimation $\sum_{i=1}^{N} \phi_{i}(0) \hat{w}_{i}(t)$, obtained from the observer that estimates the only $N$ first modes of the plant, as soon as the output is to be regulated to a non-zero value. Note that this latter estimate of the output improves as the dimension of the observer $N$ increases.

We define for $1 \leq n \leq N$ the observation error as $e_{n}(t)=$ $w_{n}(t)-\hat{w}_{n}(t)$. Hence we have

$$
\begin{aligned}
& \dot{\hat{w}}_{n}(t)=\left(-\lambda_{n}+q_{c}\right) \hat{w}_{n}(t)+a_{n} u(t)+b_{n} v(t)+l_{n} \sum_{i=1}^{N_{0}} \phi_{i}(0) e_{i}(t) \\
& +l_{n} \sum_{i=N_{0}+1}^{N} \frac{\phi_{i}(0)}{\sqrt{\lambda_{i}}} \tilde{e}_{i}(t)+l_{n} \alpha_{1} u(t)+l_{n} \zeta(t)
\end{aligned}
$$

with $\tilde{e}_{n}(t)=\sqrt{\lambda_{n}} e_{n}(t)$ and $\zeta(t)=\sum_{n \geq N+1} \phi_{n}(0) w_{n}(t)$. Hence, introducing $\hat{W}^{N_{0}}(t)=\left[\begin{array}{lll}\hat{w}_{1}(t) & \ldots & \hat{w}_{N_{0}}(t)\end{array}\right]^{\top}, E^{N_{0}}(t)=$ $\left[\begin{array}{lll}e_{1}(t) & \ldots & e_{N_{0}}(t)\end{array}\right]^{\top}, \tilde{E}^{N-N_{0}}(t)=\left[\begin{array}{lll}\tilde{e}_{N_{0}+1} & \ldots & \tilde{e}_{N}\end{array}\right]^{\top}, C_{0}=$ $\left[\begin{array}{lll}\phi_{1}(0) & \ldots & \phi_{N_{0}}(0)\end{array}\right], C_{1}=\left[\begin{array}{lll}\frac{\phi_{N_{0}+1}(0)}{\sqrt{\lambda_{N_{0}+1}}} & \ldots & \frac{\phi_{N}(0)}{\sqrt{\lambda_{N}}}\end{array}\right]$, and $L=$ $\left[\begin{array}{lll}l_{1} & \ldots & l_{N_{0}}\end{array}\right]^{\top}$, we obtain that

$$
\begin{aligned}
\dot{\hat{W}}^{N_{0}}(t)= & A_{0} \hat{W}^{N_{0}}(t)+B_{0, a} u(t)+B_{0, b} v(t)+L C_{0} E^{N_{0}}(t) \\
& +L C_{1} \tilde{E}^{N-N_{0}}(t)+\alpha_{1} L u(t)+L \zeta(t) .
\end{aligned}
$$

With

$$
\hat{W}_{a}^{N_{0}}(t)=\operatorname{col}\left(u(t), \hat{W}^{N_{0}}(t), \xi(t)\right),
$$

$\tilde{L}=\operatorname{col}(0, L, 0)$, and defining

$$
A_{1}=\left[\begin{array}{ccc}
0 & 0 & 0 \\
B_{0, a} & A_{0} & 0 \\
\alpha_{0} & C_{0} & 0
\end{array}\right], \quad B_{1}=\left[\begin{array}{c}
1 \\
B_{0, b} \\
\beta_{0}
\end{array}\right], \quad B_{r}=\left[\begin{array}{l}
0 \\
0 \\
1
\end{array}\right],
$$

we deduce that

$$
\begin{aligned}
\dot{\hat{W}}_{a}^{N_{0}}(t)= & A_{1} \hat{W}_{a}^{N_{0}}(t)+B_{1} v(t)-B_{r} r(t)+\tilde{L} C_{0} E^{N_{0}}(t) \\
& +\tilde{L} C_{1} \tilde{E}^{N-N_{0}}(t)+\alpha_{1} \tilde{L} u(t)+\tilde{L} \zeta(t)
\end{aligned}
$$

Setting the auxiliary command input as

$$
v(t)=K \hat{W}_{a}^{N_{0}}(t)
$$

and defining

$$
A_{\mathrm{cl}}\left(\alpha_{1}\right)=A_{1}+B_{1} K+\alpha_{1} \tilde{L}\left[\begin{array}{lll}
1 & 0 & 0
\end{array}\right],
$$

we obtain that

$$
\begin{aligned}
\dot{\hat{W}}_{a}^{N_{0}}(t)= & A_{\mathrm{cl}}\left(\alpha_{1}\right) \hat{W}_{a}^{N_{0}}(t)-B_{r} r(t) \\
& +\tilde{L} C_{0} E^{N_{0}}(t)+\tilde{L} C_{1} \tilde{E}^{N-N_{0}}(t)+\tilde{L} \zeta(t)
\end{aligned}
$$

and, from (8) and (14),

$$
\begin{aligned}
\dot{E}^{N_{0}}(t)= & \left(A_{0}-L C_{0}\right) E^{N_{0}}(t)-L C_{1} \tilde{E}^{N-N_{0}}(t) \\
& -\alpha_{1} L\left[\begin{array}{lll}
1 & 0 & 0
\end{array}\right] \hat{W}_{a}^{N_{0}}-L \zeta(t) .
\end{aligned}
$$

We now define $\hat{W}^{N-N_{0}}(t)=\left[\begin{array}{lll}\hat{w}_{N_{0}+1}(t) & \ldots & \hat{w}_{N}(t)\end{array}\right]^{\top}, A_{2}=$ $\operatorname{diag}\left(-\lambda_{N_{0}+1}+q_{c}, \ldots,-\lambda_{N}+q_{c}\right), B_{2, a}=\left[\begin{array}{lll}a_{N_{0}+1} & \ldots & a_{N}\end{array}\right]^{\top}$, $B_{2, b}=\left[\begin{array}{lll}b_{N_{0}+1} & \ldots & b_{N}\end{array}\right]^{\top}$. We obtain from 11 with $l_{n}=0$ for $N_{0}+1 \leq n \leq N$ that

$$
\begin{aligned}
& \dot{\hat{W}}^{N-N_{0}}(t)=A_{2} \hat{W}^{N-N_{0}}(t)+B_{2, a} u(t)+B_{2, b} v(t) \\
& =A_{2} \hat{W}^{N-N_{0}}(t)+\left(B_{2, b} K+\left[\begin{array}{lll}
B_{2, a} & 0 & 0
\end{array}\right]\right) \hat{W}_{a}^{N_{0}}(t)
\end{aligned}
$$

and, using 7b and 11,

$$
\dot{\tilde{E}}^{N-N_{0}}(t)=A_{2} \tilde{E}^{N-N_{0}}(t) .
$$

Putting now together 20.23 while introducing

$$
X(t)=\operatorname{col}\left(\hat{W}_{a}^{N_{0}}(t), E^{N_{0}}(t), \hat{W}^{N-N_{0}}(t), \tilde{E}^{N-N_{0}}(t)\right),
$$

we obtain that

$$
\dot{X}(t)=F X(t)+\mathcal{L} \zeta(t)-\mathcal{L}_{r} r(t)
$$

where

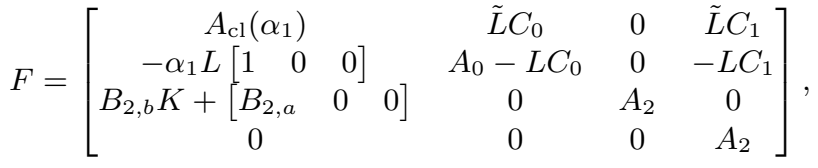

$$
\begin{aligned}
& \mathcal{L}=\operatorname{col}(\tilde{L},-L, 0,0), \quad \mathcal{L}_{r}=\operatorname{col}\left(B_{r}, 0,0,0\right) .
\end{aligned}
$$

Defining $E=\left[\begin{array}{llll}1 & 0 & \ldots & 0\end{array}\right]$ and $\tilde{K}=\left[\begin{array}{llll}K & 0 & 0 & 0\end{array}\right]$, we obtain from 15, , 18, and 24, that

$$
u(t)=E X(t), \quad v(t)=\tilde{K} X(t)
$$

and we can introduce

$$
G=\|a\|_{L^{2}}^{2} E^{\top} E+\|b\|_{L^{2}}^{2} \tilde{K}^{\top} \tilde{K} \preceq g I
$$

with $g=\|a\|_{L^{2}}^{2}+\|b\|_{L^{2}}^{2}\|K\|^{2}$ a constant independent of $N$.

Lemma 1: $\left(A_{1}, B_{1}\right)$ is controllable and $\left(A_{0}, C_{0}\right)$ is observable.

Proof. From [14, Lem. 2], $\left(A_{1}, B_{1}\right)$ is controllable if and only if

$$
\left(\left[\begin{array}{cc}
0 & 0 \\
B_{0, a} & A_{0}
\end{array}\right],\left[\begin{array}{c}
1 \\
B_{0, b}
\end{array}\right]\right)
$$

satisfies the Kalman condition and the matrix $T=$ $\left[\begin{array}{ccc}0 & 0 & 1 \\ B_{0, a} & A_{0} & B_{0, b}\end{array}\right]$ is invertible. The former condition was $\left.\begin{array}{ccc}\alpha_{0} & C_{0} & \beta_{0}\end{array}\right]$ Hence we focus on the latter one. Let $\left[\begin{array}{lllll}u_{e} & w_{1, e} & \cdots & w_{N_{0}, e} & v_{e}\end{array}\right]^{\top} \in \operatorname{ker}(T)$. We obtain that

$$
\begin{aligned}
v_{e} & =0 \\
a_{n} u_{e}+\left(-\lambda_{n}+q_{c}\right) w_{n, e} & =0, \quad 1 \leq n \leq N_{0},
\end{aligned}
$$




$$
\alpha_{0} u_{e}+\sum_{n=1}^{N_{0}} \phi_{n}(0) w_{n, e}=0 .
$$

Defining for $n \geq N_{0}+1$ the quantity $w_{n, e}=-\frac{a_{n}}{-\lambda_{n}+q_{c}} u_{e}$, we have $\left(-\lambda_{n}+q_{c}\right) w_{n, e}+a_{n} u_{e}=0$ for all $n \stackrel{\lambda_{n}+q_{c}}{\geq} 1$. Hence $\left(w_{n, e}\right)_{n \geq 1},\left(\lambda_{n} w_{n, e}\right)_{n \geq 1} \in l^{2}(\mathbb{N})$ ensuring that $w_{e} \triangleq$ $\sum_{n>1} w_{n, e} \phi_{n} \in D(\mathcal{A})$ and $\mathcal{A} w_{e}=\sum_{n>1} \lambda_{n} w_{n, e} \phi_{n}$. This shows that $-\mathcal{A} w_{e}+q_{c} w_{e}+a u_{e}=0$. Moreover, from (29c) and using (9), we infer that $w_{e}(0)=0$. From the two last identities, we have that $\left(p w_{e}^{\prime}\right)^{\prime}+\left(q_{c}-q\right) w_{e}+a u_{e}=0, w_{e}(0)=w_{e}^{\prime}(0)=0$, and $w_{e}(1)=0$. Introducing the change of variable $z_{e}(x)=w_{e}(x)+x^{2} u_{e}$, we deduce that $\left(p z_{e}^{\prime}\right)^{\prime}+\left(q_{c}-q\right) z_{e}=0, z_{e}(0)=z_{e}^{\prime}(0)=0$, and $z_{e}(1)=u_{e}$. By Cauchy uniqueness, we infer that $z_{e}=0$ hence $u_{e}=z_{e}(1)=0$. Thus we have $w_{e}=z_{e}-x^{2} u_{e}=0$ hence $w_{n, e}=0$ for all $n \geq 1$. We deduce that $\operatorname{ker}(T)=\{0\}$. Overall, we have shown that $\left(A_{1}, B_{1}\right)$ is controllable. Finally, the pair $\left(A_{0}, C_{0}\right)$ is observable because 1) $A_{0}$ is diagonal with simple eigenvalues, 2) by Cauchy uniqueness, $\phi_{n}(0) \neq 0$ for all $n \geq 1$.

In the sequel we select, once for all and independely of the dimension $N$ of the observer, the gains $K \in \mathbb{R}^{1 \times\left(N_{0}+2\right)}$ and $L \in \mathbb{R}^{N_{0}}$ so that $A_{1}+B_{1} K$ and $A_{0}-L C_{0}$ are Hurwitz with eigenvalues that have a real part strictly less than $-\delta<0$.

\section{Equilibirum condition and dynamics of deviations}

We aim at characterizing the equilibrium condition of the closedloop system composed of the reaction-diffusion system (3), the auxiliary command input dynamics $(6 \mathrm{a})$, the integral action $(10)$, the observer dynamics (11), and the state-feedback (18). To do so let $r(t)=r_{e} \in \mathbb{R}$ be arbitrary. We must solve the system of equations:

$$
\begin{aligned}
0= & \left(-\lambda_{n}+q_{c}\right) w_{n, e}+a_{n} u_{e}+b_{n} v_{e}=0, \quad n \geq 1 \\
0= & v_{e}=K \hat{W}_{a, e}^{N_{0}} \\
0= & \sum_{n=1}^{N_{0}} \phi_{n}(0) \hat{w}_{n, e}+\alpha_{0} u_{e}+\beta_{0} v_{e}-r_{e} \\
0= & \left(-\lambda_{n}+q_{c}\right) \hat{w}_{n, e}+a_{n} u_{e}+b_{n} v_{e} \\
& -l_{n}\left\{\sum_{i=1}^{N} \phi_{i}(0) \hat{w}_{i, e}-\alpha_{1} u_{e}-\tilde{y}_{e}\right\}, \quad 1 \leq n \leq N_{0} \\
0= & \left(-\lambda_{n}+q_{c}\right) \hat{w}_{n, e}+a_{n} u_{e}+b_{n} v_{e}, \quad N_{0}+1 \leq n \leq N \\
\tilde{y}_{e}= & \sum_{n \geq 1} \phi_{n}(0) w_{n, e} .
\end{aligned}
$$

We first note from $30 \mathrm{~b}$ that $v_{e}=0$. Then, from 30a we have $w_{n, e}=-\frac{a_{n}}{-\lambda_{n}+q_{c}} u_{e}$ for all $n \geq N_{0}+1$. In particular, from 30e, we have $\hat{w}_{n, e}=w_{n, e}=-\frac{a_{n}}{-\lambda_{n}+q_{c}} u_{e}$ for all $N_{0}+1 \leq n \leq N$. Detining $e_{n, e}=w_{n, e}-\hat{w}_{n, e}$ and $\zeta_{e}=\sum_{n \geq N+1} \phi_{n}(0) w_{n, e}$, we obtain that $e_{n, e}=0$ for all $N_{0}+1 \leq n \leq N$. Hence, from (30d), we infer that $0=\left(-\lambda_{n}+q_{c}\right) \hat{w}_{n, e}+a_{n} u_{e}+l_{n} \sum_{i=1}^{N_{0}} \phi_{i}(0) e_{i, e}+l_{n} \alpha_{1} u_{e}+l_{n} \zeta_{e}$ for all $1 \leq n \leq N_{0}$. Combining this latter identity with 30a, we obtain that $\left(A_{0}-L C_{0}\right) E_{e}^{N_{0}}-L \alpha_{1} u_{e}-L \zeta_{e}=0$. Invoking 12, we note that $\alpha_{1} u_{e}=-\sum_{n \geq N+1} \phi_{n}(0) w_{n, e}=-\zeta_{e}$, implying that $\left(A_{0}-L C_{0}\right) E_{e}^{N_{0}}=0$. Since $A_{0}-L C_{0}$ is Hurwitz, we infer that $e_{n, e}=0$ for all $1 \leq n<N_{0}$. In particular, $\hat{w}_{n, e}=w_{n, e}$ for all $1 \leq n \leq N$. From $30 \mathrm{~b} 30 \mathrm{~d}$ we deduce that $0=A_{\mathrm{cl}}\left(\alpha_{1}\right) \hat{W}_{a, e}^{N_{0}}-$ $B_{r} r_{e}+\tilde{L} \zeta_{e}$. Recalling that $\zeta_{e}=-\alpha_{1} u_{e}$ and $A_{\mathrm{cl}}\left(\alpha_{1}\right)$ is defined by 19], we obtain that $\left(A_{1}+B_{1} K\right) \hat{W}_{a, e}^{N_{0}}=B_{r} r_{e}$. Since $A_{1}+B_{1} K$ is Hurwitz, we infer that $\hat{W}_{a, e}^{N_{0}}=\left[\begin{array}{lllll}u_{e} & \hat{w}_{1, e} & \ldots & \hat{w}_{N_{0}, e} & \xi_{e}\end{array}\right]^{\top}=$ $\left(A_{1}+B_{1} K\right)^{-1} B_{r} r_{e}$. This is in particular compatible with $30 \mathrm{~b}$ since, based on 16, we indeed obtain that $K \hat{W}_{a, e}^{N_{0}}=0$. We note that $\left(w_{n, e}\right)_{n \geq 1},\left(\lambda_{n} w_{n, e}\right)_{n \geq 1} \in l^{2}(\mathbb{N})$ ensuring that $w_{e} \triangleq$ $\sum_{n \geq 1} w_{n, e} \phi_{n} \in D(\mathcal{A})$ and $\mathcal{A} w_{e}=\sum_{n \geq 1} \lambda_{n} w_{n, e} \phi_{n}$. Using 30a, we obtain that $-\mathcal{A} w_{e}+q_{c} w_{e}+a u_{e}+b v_{e}=0$. Introducing the change of variable $z_{e}=w_{e}+x^{2} u_{e}, z_{e}$ is a static solution of $3 \mathrm{a}$ $3 \mathrm{~b}$ associated with the constant control input $u(t)=u_{e}$. Denoting by $y_{e} \triangleq z_{e}(0)=w_{e}(0)=\tilde{y}_{e}$, we infer from 30c while invoking 9 that $r_{e}=\sum_{n=1}^{N_{0}} \phi_{n}(0) \hat{w}_{n, e}+\alpha_{0} u_{e}=\sum_{n \geq 1} \phi_{n}(0) w_{n, e}=y_{e}$. Hence, for an arbitrarily given constant reference signal $r(t)=r_{e} \in$ $\mathbb{R}$, the equilibirum condition of the closed-loop system is unique, fully characterized by $r_{e}$, and is such that $y_{e}=r_{e}$.

We can now introduce the dynamics of deviation of the different quantities w.r.t. the equilibrium condition characterized by $r_{e} \in \mathbb{R}$. In particular:

$$
\begin{aligned}
& \Delta w(t, x)=\Delta z(t, x)-x^{2} \Delta u(t), \\
& \Delta \dot{X}(t)=F \Delta X(t)+\mathcal{L} \Delta \zeta(t)-\mathcal{L}_{r} \Delta r(t), \\
& \Delta \zeta(t)=\sum_{n \geq N+1} \phi_{n}(0) \Delta w_{n}(t), \\
& \Delta \dot{w}_{n}(t)=\left(-\lambda_{n}+q_{c}\right) \Delta w_{n}(t)+a_{n} \Delta u(t)+b_{n} \Delta v(t), \\
& \Delta v(t)=K \Delta \hat{W}_{a}^{N_{0}}(t), \\
& \Delta \tilde{y}(t)=\Delta y(t)=\sum_{n \geq 1} \phi_{n}(0) \Delta w_{n}(t)
\end{aligned}
$$

with $\Delta w_{n}(t)=\left\langle\Delta w(t, \cdot), e_{n}\right\rangle$.

\section{Stability analysis and regulation assessment}

We define the constant $M_{1, \phi}=\sum_{n \geq N+1} \frac{\phi_{n}(0)^{2}}{\lambda_{n}}$, which is finite when $p \in \mathcal{C}^{2}([0,1])$ because $\phi_{n}(0)=O(1)$ as $n \rightarrow+\infty$ and $[1$ hold.

Theorem 1: Let $p \in \mathcal{C}^{2}([0,1])$ with $p>0, q \in \mathcal{C}^{0}([0,1])$ with $q \geq 0$, and $q_{c} \in \mathbb{R}$. Consider the reaction-diffusion system described by (3). Let $N_{0} \geq 1$ and $\delta>0$ be given such that $-\lambda_{n}+q_{c}<-\delta<0$ for all $n \geq N_{0}+1$. Let $K \in \mathbb{R}^{1 \times\left(N_{0}+2\right)}$ and $L \in \mathbb{R}^{N_{0}}$ be such that $A_{1}+B_{1} K$ and $A_{0}-L C_{0}$ are Hurwitz with eigenvalues that have a real part strictly less than $-\delta<0$. For a given $N \geq N_{0}+1$, assume that there exist $P \succ 0, \alpha>1$, and $\beta, \gamma>0$ such that

$$
\begin{aligned}
\Theta_{1} & =\left[\begin{array}{rr}
F^{\top} P+P F+2 \delta P+\alpha \gamma G & P \mathcal{L} \\
\mathcal{L}^{\top} P^{\top} & -\beta
\end{array}\right] \prec 0, \\
\Theta_{2} & =2 \gamma\left\{-\left(1-\frac{1}{\alpha}\right) \lambda_{N+1}+q_{c}+\delta\right\}+\beta M_{1, \phi} \leq 0 .
\end{aligned}
$$

Then, for any $\eta \in[0,1)$, there exists $M>0$ such that, for any $z_{0} \in H^{2}(0,1)$ and $u(0), \xi(0), \hat{w}_{n}(0) \in \mathbb{R}$ such that $z_{0}^{\prime}(0)=0$ and $z_{0}(1)=u(0)$, the classical solution of the closed-loop system composed of the plant (3), the integral actions (6a) and (10), the observer dynamics (11), and the state feedback (18) satisfies

$$
\begin{aligned}
& \Delta u(t)^{2}+\Delta \xi(t)^{2}+\sum_{n=1}^{N} \Delta \hat{w}_{n}(t)^{2}+\|\Delta z(t)\|_{H^{1}}^{2} \\
& \leq M e^{-2 \delta t}\left(\Delta u(0)^{2}+\Delta \xi(0)^{2}+\sum_{n=1}^{N} \Delta \hat{w}_{n}(0)^{2}+\left\|\Delta z_{0}\right\|_{H^{1}}^{2}\right) \\
& \quad+M \sup _{\tau \in[0, t]} e^{-2 \eta \delta(t-\tau)} \Delta r(\tau)^{2}
\end{aligned}
$$

for all $t \geq 0$. Moreover, the above constraints are always feasible for $N$ large enough.

Proof. Let $P \succ 0$ and $\gamma>0$ and consider the Lyapunov function candidate defined by

$$
V(\Delta X, \Delta w)=\Delta X^{\top} P \Delta X+\gamma \sum_{n \geq N+1} \lambda_{n}\left\langle\Delta w, \phi_{n}\right\rangle^{2} .
$$

with $\Delta X \in \mathbb{R}^{2 N+2}$ and $\Delta w \in D(\mathcal{A})$. The first term accounts for the dynamics of the truncated model 25, while the series, which in view of 2 is related to the $H^{1}$ norm of the PDE trajectories, is used to handle the modes $w_{n}$ for $n \geq N+1$ of the PDE plant. Proceeding 
exactly as in [13] but taking into account the extra contribution of the reference signal appearing in 25, we obtain for $t \geq 0$ that

$$
\begin{aligned}
& \dot{V}(t)+2 \delta V(t) \leq\left[\begin{array}{c}
\Delta X(t) \\
\Delta \zeta(t)
\end{array}\right]^{\top} \Theta_{1}\left[\begin{array}{c}
\Delta X(t) \\
\Delta \zeta(t)
\end{array}\right] \\
& -2 \Delta X(t)^{\top} P \mathcal{L}_{r} \Delta r(t)+\sum_{n \geq N+1} \lambda_{n} \Gamma_{n} \Delta w_{n}(t)^{2}
\end{aligned}
$$

with $\Gamma_{n}=2 \gamma\left\{-\left(1-\frac{1}{\alpha}\right) \lambda_{n}+q_{c}+\delta\right\}+\beta M_{1, \phi}$ for $n \geq N+1$, $\alpha>1$ and $\beta>0$ arbitrary, and where, with a slight abuse of notation, $\dot{V}(t)$ denotes the time derivative of $V(X(t), w(t))$ along the system trajectories (31). Since $\alpha>1$ we have $\Gamma_{n} \leq \Theta_{2} \leq 0$ for all $n \geq N+1$. From 32a), there exists $\epsilon>0$ such that $\Theta_{1} \preceq-\epsilon I$. Hence the assumptions imply that $\dot{V}(t)+2 \delta V(t) \leq-\epsilon\|\Delta X(t)\|^{2}-$ $2 \Delta X(t)^{\top} P \mathcal{L}_{r} \Delta r(t) \leq \frac{\left\|P \mathcal{L}_{r}\right\|^{2}}{\epsilon} \Delta r(t)^{2}$ where Young's inequality has been used to derive the latter estimate. After integration, we obtain for any $\eta \in[0,1)$ the existence of a constant $M_{1}>0$ such that $V(t) \leq e^{-2 \delta t} V(0)+M_{1} \sup _{\tau \in[0, t]} e^{-2 \eta \delta(t-\tau)} \Delta r(\tau)^{2}$ for all $t \geq 0$. The claimed estimate (33) easily follows from the definition (34) of the Lyapunov function, the use of 2], Poincaré's inequality, and the change of variable $31 \mathrm{a}$.

We now show that we can always select $N \geq N_{0}+1, P \succ 0$, $\alpha>1$, and $\beta, \gamma>0$ such that 32 holds. By the Schur complement, $\Theta_{1} \prec 0$ is equivalent to $F^{\top} P+P F+2 \delta P+\alpha \gamma G+\frac{1}{\beta} P \mathcal{L} \mathcal{L}^{\top} P^{\top} \prec 0$. We define $F=F_{1}+F_{2}$ where

$$
\begin{aligned}
& F_{1}=\left[\begin{array}{ccccc}
A_{1}+B_{1} K & \tilde{L} C_{0} & 0 & \tilde{L} C_{1} \\
0 & & A_{0}-L C_{0} & 0 & -L C_{1} \\
B_{2, b} K+\left[\begin{array}{ccc}
B_{2, a} & 0 & 0
\end{array}\right] & 0 & A_{2} & 0 \\
0 & & 0 & 0 & A_{2}
\end{array}\right],
\end{aligned}
$$

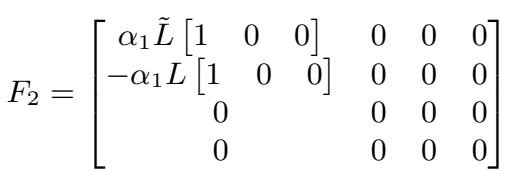

with $\left\|F_{2}\right\| \rightarrow 0$, because $\alpha_{1} \rightarrow 0$, when $N \rightarrow+\infty$. We note that $A_{1}+B_{1} K+\delta I$ and $A_{0}-L C_{0}+\delta I$ are Hurwitz while $\left\|e^{\left(A_{2}+\delta I\right) t}\right\| \leq e^{-\kappa_{0} t}$ with $\kappa_{0}=\lambda_{N_{0}+1}-q_{c}-\delta>0$. Moreover, $\left\|\tilde{L} C_{1}\right\| \leq\|L\|\left\|C_{1}\right\|,\left\|L C_{1}\right\| \leq\|L\|\left\|C_{1}\right\|$, with $\left\|C_{1}\right\|=O(1)$ as $N \rightarrow+\infty$ while $\left\|B_{2, b} K+\left[\begin{array}{ccc}B_{2, a} & 0 & 0\end{array}\right]\right\| \leq\|b\|_{L^{2}}\|K\|+\|a\|_{L^{2}}$ where the right-hand side is a constant independent of $N$. The application of [13 Lemma in Appendix], which is a generalization of a result found in [11], to the matrix $F_{1}+\delta I$ gives the existence of $P \succ 0$ such that $F_{1} P+P F_{1}+2 \delta P=-I$ and $\|P\|=O(1)$ as $N \rightarrow+\infty$. Therefore, we have $F^{\top} P+P F+2 \delta P+\alpha \gamma G+$ $\frac{1}{\beta} P \mathcal{L} \mathcal{L}^{\top} P^{\top}=-I+F_{2}^{\top} P+P F_{2}+\alpha \gamma G+\frac{1}{\beta} P \mathcal{L} \mathcal{L}^{\top} P^{\top}$ where $G$ satisfies 27, and $\|\mathcal{L}\|=\sqrt{2}\|L\|$, which is independent of $N$. Hence, fixing arbitrarily $\alpha>1$ while setting $\beta=\sqrt{N}$ and $\gamma=N^{-1}$, we infer that (32) holds for $N \geq N_{0}+1$ large enough.

Remark 1: Constraints 32 are nonlinear w.r.t. the decision variables. However, for a given value of $N \geq N_{0}+1$ and arbitrarily fixing $\alpha>1$, constraints 32 become LMI conditions w.r.t. the decision variables $P \succ 0$ and $\beta, \gamma>0$. As shown in the proof of Theorem 1. these latter LMI conditions are always feasible provided the order of the observer $N$ is selected large enough. Similar remarks apply to Theorems 3 and 5

We now assess the setpoint regulation of the left Dirichlet trace.

Theorem 2: Under both assumptions and conclusions of Theorem 1 for any $\eta \in[0,1)$, there exists $M_{r}>0$ such that

$$
\begin{gathered}
|y(t)-r(t)| \leq M_{r} e^{-\delta t}\left(|\Delta u(0)|+|\Delta \xi(0)|+\sum_{n=1}^{N}\left|\Delta \hat{w}_{n}(0)\right|\right. \\
\left.+\left\|\Delta z_{0}\right\|_{H^{1}}\right)+M_{r} \sup _{\tau \in[0, t]} e^{-\eta \delta(t-\tau)}|\Delta r(\tau)|
\end{gathered}
$$

for all $t \geq 0$.

Proof. Recalling that $y_{e}=r_{e}$, one has $|y(t)-r(t)| \leq|\Delta y(t)|+$ $|\Delta r(t)|$. From 31f] and Cauchy-Schwarz inequality, we infer that $|\Delta y(t)| \leq \sqrt{\sum_{n \geq 1} \frac{\phi_{n}(0)^{2}}{\lambda_{n}}} \sqrt{\sum_{n \geq 1} \lambda_{n} \Delta w_{n}(t)^{2}}$. Using now 22 we infer the existence of a constant $M_{2}>0$ such that $|\Delta y(t)| \leq$ $M_{2}\|\Delta w(t)\|_{H^{1}}$. The proof is completed by invoking the change of variable $31 \mathrm{a}$ and the stability result 33 .

\section{NEUMANN MEASUREMENT AND REGULATION CONTROL}

We now consider the reaction-diffusion system with Neumann boundary observation described for $t>0$ and $x \in(0,1)$ by

$$
\begin{aligned}
z_{t}(t, x) & =\left(p(x) z_{x}(t, x)\right)_{x}+\left(q_{c}-q(x)\right) z(t, x) \\
z(t, 0) & =0, \quad z(t, 1)=u(t) \\
z(0, x) & =z_{0}(x) \\
y(t) & =z_{x}(t, 0)
\end{aligned}
$$

in the case $p \in \mathcal{C}^{2}([0,1])$.

\section{A. Control design}

Introducing the change of variable

$$
w(t, x)=z(t, x)-x u(t)
$$

we obtain

$$
\begin{aligned}
& w_{t}(t, x)=\left(p(x) w_{x}(t, x)\right)_{x}+\left(q_{c}-q(x)\right) w(t, x) \\
& +a(x) u(t)+b(x) \dot{u}(t) \\
& w(t, 0)=0, \quad w(t, 1)=0 \\
& w(0, x)=w_{0}(x) \\
& \tilde{y}(t)=w_{x}(t, 0)
\end{aligned}
$$

with $a, b \in L^{2}(0,1)$ defined by $a(x)=p^{\prime}(x)+\left(q_{c}-q(x)\right) x$ and $b(x)=-x$, respectively, $\tilde{y}(t)=y(t)-u(t)$, and $w_{0}(x)=z_{0}(x)-$ $x u(0)$. Introducing the auxiliary command input $v(t)=\dot{u}(t)$, we infer that (6) still holds but the domain of $\mathcal{A}$ is now replaced by $D(\mathcal{A})=\left\{f \in H^{2}(0,1): f(0)=f(1)=0\right\}$. Then, considering classical solutions associated with any $z_{0} \in H^{2}(0,1)$ and any $u(0) \in$ $\mathbb{R}$ such that $z_{0}(0)=0$ and $z_{0}(1)=u(0)$ (their existence for the upcoming closed-loop dynamics is an immediate consequence of [19. Chap. 6, Thm. 1.7]), 7a 7b is still valid while $7 \mathrm{c}$ is replaced by

$$
\tilde{y}(t)=\sum_{i \geq 1} \phi_{i}^{\prime}(0) w_{i}(t) \text {. }
$$

Based on similar motivations than the ones reported in Section III. we consider the integral component

$$
\dot{\xi}(t)=\sum_{n=1}^{N_{0}} \phi_{n}^{\prime}(0) \hat{w}_{n}(t)+\alpha_{0} u(t)+\beta_{0} v(t)-r(t) .
$$

with

$$
\alpha_{0}=1-\sum_{n \geq N_{0}+1} \frac{a_{n} \phi_{n}^{\prime}(0)}{-\lambda_{n}+q_{c}}, \quad \beta_{0}=-\sum_{n \geq N_{0}+1} \frac{b_{n} \phi_{n}^{\prime}(0)}{-\lambda_{n}+q_{c}}
$$

and where the observation dynamics, for $1 \leq n \leq N$, take the form:

$$
\begin{aligned}
\dot{\hat{w}}_{n}(t)= & \left(-\lambda_{n}+q_{c}\right) \hat{w}_{n}(t)+a_{n} u(t)+b_{n} v(t) \\
& -l_{n}\left(\sum_{i=1}^{N} \phi_{i}^{\prime}(0) \hat{w}_{i}(t)-\alpha_{1} u(t)-\tilde{y}(t)\right)
\end{aligned}
$$

with

$$
\alpha_{1}=\sum_{n \geq N+1} \frac{a_{n} \phi_{n}^{\prime}(0)}{-\lambda_{n}+q_{c}}
$$


and where $l_{n} \in \mathbb{R}$ are the observer gains. We set $l_{n}=0$ for $N_{0}+1 \leq n \leq N$. Proceeding now as in Section III but with the updated versions of the matrices $C_{0}$ and $C_{1}$ now given by $C_{0}=$ $\left[\begin{array}{llll}\phi_{1}^{\prime}(0) & \ldots & \phi_{N_{0}}^{\prime}(0)\end{array}\right]$ and $C_{1}=\left[\begin{array}{lll}\frac{\phi_{N_{0}+1}^{\prime}(0)}{\lambda_{N_{0}+1}} & \ldots & \frac{\phi_{N}^{\prime}(0)}{\lambda_{N}}\end{array}\right]$ while redefining $\tilde{e}_{n}(t)$ and $\zeta(t)$ as $\tilde{e}_{n}(t) \stackrel{\lambda_{0}+1}{=} \lambda_{n} e_{n}(t)$ and $\zeta(t) \stackrel{ }{=}$ $\sum_{n \geq N+1} \phi_{n}^{\prime}(0) w_{n}(t)$, we infer that 25 holds.

Lemma 2: $\left(A_{1}, B_{1}\right)$ is controllable and $\left(A_{0}, C_{0}\right)$ is observable.

The proof of this Lemma is analogous to the one of Lemma 1 and is thus omitted. We select in the sequel $K \in \mathbb{R}^{1 \times\left(N_{0}+2\right)}$ and $L \in \mathbb{R}^{N_{0}}$ such that $A_{1}+B_{1} K$ and $A_{0}-L C_{0}$ are Hurwitz.

\section{B. Equilibirum condition and dynamics of deviations}

Proceeding similarly to Section III we can characterize the equilibrium condition of the closed-loop system composed of the reactiondiffusion system 38, the auxiliary command input dynamics 6a, the integral action (42), the observer dynamics (44, and the statefeedback 18). In particular, setting $r(t)=r_{e} \in \mathbb{R}$, it can be shown that there exists a unique solution to:

$$
\begin{aligned}
0= & \left(-\lambda_{n}+q_{c}\right) w_{n, e}+a_{n} u_{e}+b_{n} v_{e}=0, \quad n \geq 1 \\
0= & v_{e}=K \hat{W}_{a, e}^{N_{0}} \\
0= & \sum_{n=1}^{N_{0}} \phi_{n}^{\prime}(0) \hat{w}_{n, e}+\alpha_{0} u_{e}+\beta_{0} v_{e}-r_{e} \\
0= & \left(-\lambda_{n}+q_{c}\right) \hat{w}_{n, e}+a_{n} u_{e}+b_{n} v_{e} \\
& -l_{n}\left\{\sum_{i=1}^{N} \phi_{i}^{\prime}(0) \hat{w}_{i, e}-\alpha_{1} u_{e}-\tilde{y}_{e}\right\}, \quad 1 \leq n \leq N_{0}, \\
0= & \left(-\lambda_{n}+q_{c}\right) \hat{w}_{n, e}+a_{n} u_{e}+b_{n} v_{e}, \quad N_{0}+1 \leq n \leq N, \\
\tilde{y}_{e}= & \sum_{n \geq 1} \phi_{n}^{\prime}(0) w_{n, e} .
\end{aligned}
$$

Moreover we can define $w_{e} \triangleq \sum_{n \geq 1} w_{n, e} \phi_{n} \in D(\mathcal{A})$. Introducing the change of variable $z_{e}=w_{e}+x u_{e}, z_{e}$ is a static solution of 38a $38 \mathrm{~b}$ associated with the constant control input $u(t)=u_{e}$. Denoting by $y_{e} \triangleq z_{e}^{\prime}(0)$, we also infer that $y_{e}=r_{e}$, achieving the desired reference tracking. This allows the introduction of the dynamics of deviation of the different quantities w.r.t. the equilibrium condition characterized by $r_{e} \in \mathbb{R}$. We have:

$$
\begin{aligned}
& \Delta w(t, x)=\Delta z(t, x)-x \Delta u(t), \\
& \Delta \dot{X}(t)=F \Delta X(t)+\mathcal{L} \Delta \zeta(t)-\mathcal{L}_{r} \Delta r(t), \\
& \Delta \zeta(t)=\sum_{n \geq N+1} \phi_{n}^{\prime}(0) \Delta w_{n}(t), \\
& \Delta \dot{w}_{n}(t)=\left(-\lambda_{n}+q_{c}\right) \Delta w_{n}(t)+a_{n} \Delta u(t)+b_{n} \Delta v(t), \\
& \Delta v(t)=K \Delta \hat{W}_{a}^{N_{0}}(t), \\
& \Delta \tilde{y}(t)=\Delta y(t)-\Delta u(t)=\sum_{n \geq 1} \phi_{n}^{\prime}(0) \Delta w_{n}(t) .
\end{aligned}
$$

\section{Stability analysis and regulation assessment}

We define, for any $\epsilon \in(0,1 / 2]$, the constant $M_{2, \phi}(\epsilon)=$ $\sum_{n \geq N+1} \frac{\phi_{n}^{\prime}(0)^{2}}{\lambda_{n}^{3 / 2+\epsilon}}$, which is finite when $p \in \mathcal{C}^{2}([0,1])$ because we recall that $\phi_{n}^{\prime}(0)=O\left(\sqrt{\lambda_{n}}\right)$ as $n \rightarrow+\infty$ and $\sqrt{1}$ hold.

Theorem 3: Let $p \in \mathcal{C}^{2}([0,1])$ with $p>0, q \in \mathcal{C}^{0}([0,1])$ with $q \geq 0$, and $q_{c} \in \mathbb{R}$. Consider the reaction-diffusion system described by (38). Let $N_{0} \geq 1$ and $\delta>0$ be given such that $-\lambda_{n}+q_{c}<-\delta<$ 0 for all $n \geq N_{0}+1$. Let $K \in \mathbb{R}^{1 \times\left(N_{0}+2\right)}$ and $L \in \mathbb{R}^{N_{0}}$ be such that $A_{1}+B_{1} K$ and $A_{0}-L C_{0}$ are Hurwitz with eigenvalues that have a real part strictly less than $-\delta<0$. For a given $N \geq N_{0}+1$, assume that there exist $P \succ 0, \epsilon \in(0,1 / 2], \alpha>1$, and $\beta, \gamma>0$ such that $\Theta_{1} \prec 0$, where $\Theta_{1}$ is defined by $32 a$,

$$
\begin{aligned}
& \Theta_{2}=2 \gamma\left\{-\left(1-\frac{1}{\alpha}\right) \lambda_{N+1}+q_{c}+\delta\right\}+\beta M_{2, \phi}(\epsilon) \lambda_{N+1}^{1 / 2+\epsilon} \leq 0, \\
& \Theta_{3}=2 \gamma\left(1-\frac{1}{\alpha}\right)-\frac{\beta M_{2, \phi}(\epsilon)}{\lambda_{N+1}^{1 / 2-\epsilon}} \geq 0 .
\end{aligned}
$$

Then, for any $\eta \in[0,1)$, there exists $M>0$ such that, for any $z_{0} \in H^{2}(0,1)$ and $u(0), \xi(0), \hat{w}_{n}(0) \in \mathbb{R}$ such that $z_{0}(0)=0$ and $z_{0}(1)=u(0)$, the classical solution of the closed-loop system composed of the plant (38), the integral actions (6a) and (42), the observer dynamics (44), and the state feedback (18) satisfies (33) for all $t \geq 0$. Moreover, the above constraints are always feasible for $N$ large enough.

Proof. Let $P \succ 0$ and $\gamma>0$ and consider the Lyapunov function candidate defined by 34. Then, proceeding as in 13 but taking into account the extra contribution of the reference signal appearing in 25, we obtain that (35) holds for all $t \geq 0$ with $\Gamma_{n}=2 \gamma\left\{-\left(1-\frac{1}{\alpha}\right) \lambda_{n}+q_{c}+\delta\right\}+\beta M_{2, \phi}(\epsilon) \lambda_{n}^{1 / 2+\epsilon}$. Since $\epsilon \in(0,1 / 2]$, we have $\lambda_{n}^{1 / 2+\epsilon}=\lambda_{n} / \lambda_{n}^{1 / 2-\epsilon} \leq \lambda_{n} / \lambda_{N+1}^{1 / 2-\epsilon}$ for all $n \geq N+1$, hence $\Gamma_{n} \leq-\Theta_{3} \lambda_{n}+2 \gamma\left\{q_{c}+\delta\right\} \leq \Theta_{2} \leq 0$ for all $n \geq N+1$ where we have used that $\Theta_{3} \geq 0$. Now the proof of the stability estimate (33) is analogous to the one reported in the proof of Theorem 1

It remains to show that we can always select $N \geq N_{0}+1, P \succ 0$, $\epsilon \in(0,1 / 2], \alpha>1$, and $\beta, \gamma>0$ such that $\Theta_{1} \prec 0, \Theta_{2} \leq 0$, and $\Theta_{3} \geq 0$. To handle the constraint $\Theta_{1} \prec 0$, we proceed as in the last part of the proof of Theorem 1 This is allowed because $\left\|C_{1}\right\|=O(1)$ as $N \rightarrow+\infty$. We set $\epsilon=1 / 8$ and we arbitrary fix $\alpha>1$. Setting $\beta=N^{1 / 8}$ and $\gamma=N^{-3 / 16}$, we deduce the existence of an integer $N \geq N_{0}+1$ large enough such that $\Theta_{1} \prec 0, \Theta_{2} \leq 0$, and $\Theta_{3} \geq 0$.

We are now in position to assess the setpoint regulation control of the left Dirichlet trace.

Theorem 4: Under both assumptions and conclusions of Theorem 3. for any $\eta \in[0,1)$, there exists $M_{r}>0$ such that

$$
\begin{aligned}
& |y(t)-r(t)| \leq M_{r} e^{-\delta t}\left(|\Delta u(0)|+|\Delta \xi(0)|+\sum_{n=1}^{N}\left|\Delta \hat{w}_{n}(0)\right|\right. \\
& \left.+\left\|\Delta z_{0}\right\|_{H^{1}}+\left\|\mathcal{A} \Delta w_{0}\right\|_{L^{2}}\right)+M_{r} \sup _{\tau \in[0, t]} e^{-\eta \delta(t-\tau)}|\Delta r(\tau)|
\end{aligned}
$$

for all $t \geq 0$ where $\Delta w_{0}=\Delta z_{0}-x \Delta u(0)$.

Proof. Recalling that $y_{e}=r_{e}$, one has $|y(t)-r(t)| \leq|\Delta y(t)|+$ $|\Delta r(t)|$. We infer from (47f) and Cauchy-Schwarz inequality that $|\Delta y(t)| \leq \sqrt{\sum_{n \geq 1} \frac{\phi_{n}^{\prime}(0)^{2}}{\lambda_{n}^{2}}} \sqrt{\sum_{n \geq 1} \lambda_{n}^{2} \Delta w_{n}(t)^{2}}+|\Delta u(t)|$. In view of the stability estimate (33) provided by Theorem 3 , we only need to study the term $\sum_{n>1} \lambda_{n}^{2} \Delta w_{n}(t)^{2}$. This can be done as in 14 Proof of Theorem 2], yielding the claimed estimate 48.

\section{Dirichlet measurement and Neumann Regulation CONTROL}

We now consider the reaction-diffusion system described by $3 \mathrm{a}$ $3 \mathrm{c}$, still in the case $p \in \mathcal{C}^{2}([0,1])$, but this time with the boundary measurement $y_{m}(t)$ and the distinct and unmeasured output to-beregulated $y_{r}(t)$ described by:

$$
y_{m}(t)=z(t, 0), \quad y_{r}(t)=z_{x}(t, 1) .
$$

\section{A. Control design}

Using the change of variable (4), we obtain that 5a 5c still hold while [5d] is replaced by

$$
\tilde{y}_{m}(t)=w(t, 0)=z(t, 0)=y_{m}(t),
$$




$$
\tilde{y}_{r}(t)=w_{x}(t, 1)=z_{x}(t, 1)-2 u(t)=y_{r}(t)-2 u(t) .
$$

Then, considering classical solutions, $7 \mathrm{a} 7 \mathrm{~b}$ is still valid while $7 \mathrm{c}$ is replaced by

$$
\tilde{y}_{m}(t)=\sum_{i \geq 1} \phi_{i}(0) w_{i}(t), \quad \tilde{y}_{r}(t)=\sum_{i \geq 1} \phi_{i}^{\prime}(1) w_{i}(t) .
$$

Based on similar motivations that the ones reported in Section III we consider the integral component

$$
\dot{\xi}(t)=\sum_{n=1}^{N_{0}} \phi_{n}^{\prime}(1) \hat{w}_{n}(t)+\alpha_{0} u(t)+\beta_{0} v(t)-r(t) .
$$

with

$$
\alpha_{0}=2-\sum_{n \geq N_{0}+1} \frac{a_{n} \phi_{n}^{\prime}(1)}{-\lambda_{n}+q_{c}}, \quad \beta_{0}=-\sum_{n \geq N_{0}+1} \frac{b_{n} \phi_{n}^{\prime}(1)}{-\lambda_{n}+q_{c}}
$$

and where the observation dynamics, for $1 \leq n \leq N$, takes the form:

$$
\begin{aligned}
\dot{\hat{w}}_{n}(t)= & \left(-\lambda_{n}+q_{c}\right) \hat{w}_{n}(t)+a_{n} u(t)+b_{n} v(t) \\
& -l_{n}\left(\sum_{i=1}^{N} \phi_{i}(0) \hat{w}_{i}(t)-\alpha_{1} u(t)-\tilde{y}_{m}(t)\right)
\end{aligned}
$$

with

$$
\alpha_{1}=\sum_{n \geq N+1} \frac{a_{n} \phi_{n}(0)}{-\lambda_{n}+q_{c}}
$$

and where $l_{n} \in \mathbb{R}$ are the observer gains. We set $l_{n}=0$ for $N_{0}+1 \leq n \leq N$. Adopting now the same definitions as the ones used in Section III except that the matrix $A_{1}$, originally defined by 16 , is now replaced by $A_{1}=\left[\begin{array}{ccc}0 & 0 & 0 \\ B_{0, a} & A_{0} & 0 \\ \alpha_{0} & C_{r} & 0\end{array}\right]$ where $C_{r}=\left[\begin{array}{lll}\phi_{1}^{\prime}(1) & \ldots & \phi_{N_{0}}^{\prime}(1)\end{array}\right]$, we infer that 25 holds.

Lemma 3: The pair $\left(A_{0}, C_{0}\right)$ is observable. If the unique solution of $\left(p f^{\prime}\right)^{\prime}+\left(q_{c}-q\right) f=0$ with $f(1)=1$ and $f^{\prime}(1)=0$ is such that $f^{\prime}(0) \neq 0$, then the pair $\left(A_{1}, B_{1}\right)$ is controllable.

Proof. The observability of $\left(A_{0}, C_{0}\right)$ was assessed in Lemma 11 From [14, Lem. 2], and because the pair 28 is controllable, then $\left(A_{1}, B_{1}\right)$ is controllable if and only if the matrix $T=\left[\begin{array}{ccc}0 & 0 & 1 \\ B_{0, a} & A_{0} & B_{0, b} \\ \alpha_{0} & C_{r} & \beta_{0}\end{array}\right]$ is invertible. Let $\left[\begin{array}{lllll}u_{e} & w_{1, e} & \ldots & w_{N_{0}, e} & v_{e}\end{array}\right]^{\top} \in \operatorname{ker}(T)$. We obtain that $v_{e}=0, a_{n} u_{e}+\left(-\lambda_{n}+q_{c}\right) w_{n, e}=0$ for all $1 \leq n \leq N_{0}$, and $\alpha_{0} u_{e}+\sum_{n=1}^{N_{0}} \phi_{n}^{\prime}(1) w_{n, e}=0$. Defining for $n \geq N_{0}+1$ the quantity $w_{n, e}=-\frac{a_{n}}{-\lambda_{n}+q_{c}} u_{e}$, we have $\left(-\lambda_{n}+q_{c}\right) w_{n, e}+a_{n} u_{e}=0$ for all $n \geq 1$. Hence $\left(w_{n, e}\right)_{n \geq 1},\left(\lambda_{n} w_{n, e}\right)_{n \geq 1} \in l^{2}(\mathbb{N})$ ensuring that $w_{e} \triangleq \sum_{n \geq 1} w_{n, e} \phi_{n} \in D(\mathcal{A})$ and $\mathcal{A} w_{e}=\sum_{n \geq 1} \lambda_{n} w_{n, e} \phi_{n}$. This shows that $-\mathcal{A} w_{e}+q_{c} w_{e}+a u_{e}=0$. Moreover, using (53), we also have $0=\alpha_{0} u_{e}+\sum_{n=1}^{N_{0}} \phi_{n}^{\prime}(1) w_{n, e}=2 u_{e}+w_{e}^{\prime}(1)$. From the two latter identities, we infer that $\left(p w_{e}^{\prime}\right)^{\prime}+\left(q_{c}-q\right) w_{e}+a u_{e}=0$, $w_{e}^{\prime}(0)=w_{e}(1)=0$, and $w_{e}^{\prime}(1)+2 u_{e}=0$. Introducing the change of variable $z_{e}(x)=w_{e}(x)+x^{2} u_{e}$, we deduce that $\left(p z_{e}^{\prime}\right)^{\prime}+\left(q_{c}-q\right) z_{e}=0, z_{e}^{\prime}(0)=z_{e}^{\prime}(1)=0$, and $z_{e}(1)=u_{e}$. From our assumption, we infer that $u_{e}=0$ and $z_{e}=0$. Thus we have $w_{e}=z_{e}-x^{2} u_{e}=0$ hence $w_{n, e}=0$ for all $n \geq 1$. We deduce that $\operatorname{ker}(T)=\{0\}$, showing that $\left(A_{1}, B_{1}\right)$ is controllable.

We select $K \in \mathbb{R}^{1 \times\left(N_{0}+2\right)}$ and $L \in \mathbb{R}^{N_{0}}$ so that $A_{1}+B_{1} K$ and $A_{0}-L C_{0}$ are Hurwitz.

\section{B. Equilibrium condition and dynamics of deviations}

Proceeding as in Section III we can characterize the equilibrium condition of the closed-loop system composed of the reactiondiffusion system 3a 3c with 49, the auxiliary command input dynamics 6a, the integral action (52, the observer dynamics (54), and the state-feedback (18). In particular, setting $r(t)=r_{e} \in \mathbb{R}$, it can be shown that there exists a unique solution to :

$$
\begin{aligned}
0= & \left(-\lambda_{n}+q_{c}\right) w_{n, e}+a_{n} u_{e}+b_{n} v_{e}=0, \quad n \geq 1, \\
0= & v_{e}=K \hat{W}_{a, e}^{N_{0}}, \\
0= & \sum_{n=1}^{N_{0}} \phi_{n}^{\prime}(1) \hat{w}_{n, e}+\alpha_{0} u_{e}+\beta_{0} v_{e}-r_{e}, \\
0= & \left(-\lambda_{n}+q_{c}\right) \hat{w}_{n, e}+a_{n} u_{e}+b_{n} v_{e} \\
& -l_{n}\left\{\sum_{i=1}^{N} \phi_{i}(0) \hat{w}_{i, e}-\alpha_{1} u_{e}-\tilde{y}_{e}\right\}, 1 \leq n \leq N_{0}, \\
0= & \left(-\lambda_{n}+q_{c}\right) \hat{w}_{n, e}+a_{n} u_{e}+b_{n} v_{e}, \quad N_{0}+1 \leq n \leq N,
\end{aligned}
$$

$$
\tilde{y}_{m, e}=\sum_{n \geq 1} \phi_{n}(0) w_{n, e}, \quad \tilde{y}_{r, e}=\sum_{n \geq 1} \phi_{n}^{\prime}(1) w_{n, e} .
$$

Moreover we can define $w_{e} \triangleq \sum_{n \geq 1} w_{n, e} \phi_{n} \in D(\mathcal{A})$. Introducing the change of variable $z_{e}=w_{e}+x^{2} u_{e}, z_{e}$ is a static solution of $3 \mathrm{a}$. $3 \mathrm{~b}$ associated with the constant control input $u(t)=u_{e}$. Denoting by $y_{r, e} \triangleq z_{e}^{\prime}(1)$, we also infer that $y_{r, e}=r_{e}$, achieving the desired reference tracking. Consequently, we obtain the following dynamics of deviations:

$$
\begin{aligned}
\Delta w(t, x) & =\Delta z(t, x)-x^{2} \Delta u(t), \\
\Delta \dot{X}(t) & =F \Delta X(t)+\mathcal{L} \Delta \zeta(t)-\mathcal{L}_{r} \Delta r(t), \\
\Delta \zeta(t) & =\sum_{n \geq N+1} \phi_{n}(0) \Delta w_{n}(t), \\
\Delta \dot{w}_{n}(t) & =\left(-\lambda_{n}+q_{c}\right) \Delta w_{n}(t)+a_{n} \Delta u(t)+b_{n} \Delta v(t), \\
\Delta v(t) & =K \Delta \hat{W}_{a}^{N_{0}}(t), \\
\Delta \tilde{y}_{m}(t) & =\Delta y_{m}(t)=\sum_{n \geq 1} \phi_{n}(0) \Delta w_{n}(t), \\
\Delta \tilde{y}_{r}(t) & =\Delta y_{r}(t)-2 \Delta u(t)=\sum_{n \geq 1} \phi_{n}^{\prime}(1) \Delta w_{n}(t) .
\end{aligned}
$$

\section{Stability analysis and regulation assessment}

The proof of the following theorem directly follows from the proofs reported in the previous sections.

Theorem 5: Under the assumption of Lemma 3, the stability result stated by Theorem 11 also applies to the closed-loop system composed of the plant (3a 3c) with (49), the integral actions (6a) and (52), the observer dynamics (54), and the state feedback (18). Moreover, for any $\eta \in[0,1)$, there exists $M_{r}>0$ such that

$$
\begin{aligned}
& \left|y_{r}(t)-r(t)\right| \leq M_{r} e^{-\delta t}\left(|\Delta u(0)|+|\Delta \xi(0)|+\sum_{n=1}^{N}\left|\Delta \hat{w}_{n}(0)\right|\right. \\
& \left.+\left\|\Delta z_{0}\right\|_{H^{1}}+\left\|\mathcal{A} \Delta w_{0}\right\|_{L^{2}}\right)+M_{r} \sup _{\tau \in[0, t]} e^{-\eta \delta(t-\tau)}|\Delta r(\tau)|
\end{aligned}
$$

for all $t \geq 0$ where $\Delta w_{0}=\Delta z_{0}-x^{2} \Delta u(0)$.

\section{NUMERICAL ILLUSTRATION}

We illustrate the result of Section $\square$ for Dirichlet measurement and Neumann regulation using a modal approximation that captures the 50 dominant modes of the reaction-diffusion PDE. We set $p=1, q=0$, and $q_{c}=3$ for which the open-loop plant is unstable. Selecting $\delta=0.5$, we obtain $N_{0}=1$, the feedback gain $K=\left[\begin{array}{lll}-10.4134 & -11.3747 & 2.3100\end{array}\right]$, and the observer gain $L=1.4373$. The conditions of Theorem 5 are found feasible for $N=3$. The time-domain evolution of the closed-loop system trajectories are depicted in Fig. 1 confirming the theoretical predictions. 


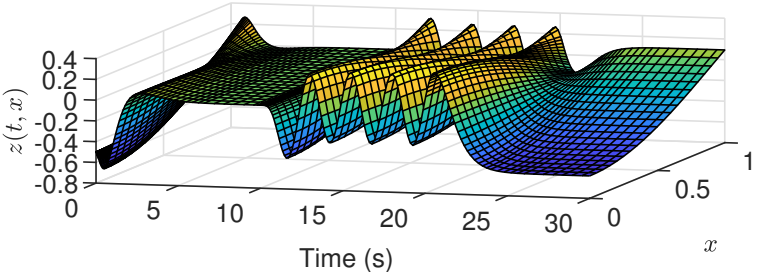

(a) State $z(t, x)$

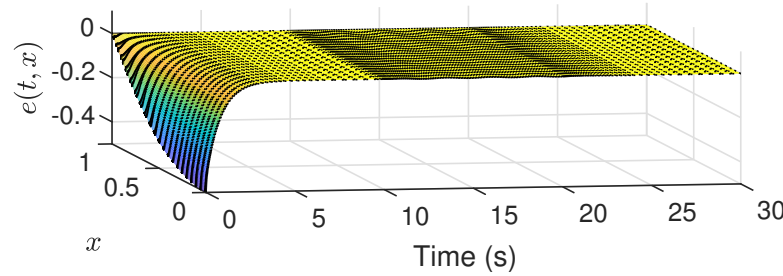

(b) Observation error $e(t, x)=w(t, x)-\sum_{n=1}^{N} \hat{w}_{n}(t) \phi_{n}(x)$

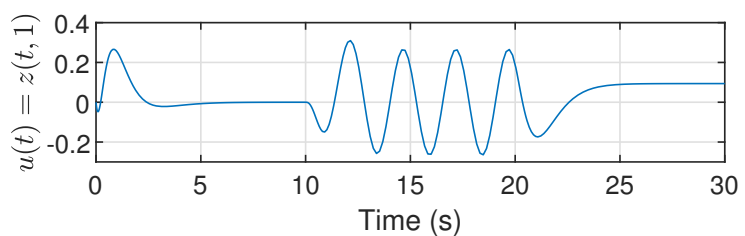

(c) Command input $u(t)=z(t, 1)$

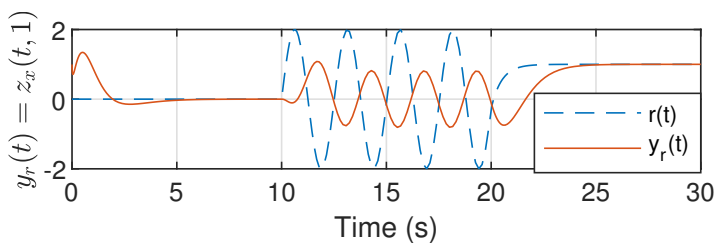

(d) Regulated output $y_{r}(t)=z_{x}(t, 1)$

Fig. 1. Time evolution in closed-loop with Dirichlet boundary measurement $y_{m}(t)=z(t, 0)$ and Neumann boundary regulation $y_{r}(t)=z_{x}(t, 1)$ for the reaction-diffusion system $3 \mathrm{a} \mid 3 \mathrm{c}$

\section{CONCLUSION}

We proposed the design of a finite-dimensional observer-based PI controller to achieve the both output stabilization and regulation control of reaction-diffusion PDEs. Even if presented for a Dirichlet boundary control input, the presented results easily extend to Neumann/Robin boundary control (by modifying the change of variable formula to obtain an homogeneous PDE, giving different $\left.a, b \in L^{2}(0,1)\right)$. In-domain measurements can also be handled with the same approach provided the satisfaction of adequate observability conditions. While we have adopted in this paper an early lumping approach, future research directions for finite-dimensional PI regulation control of reaction-diffusion PDEs may be concerned with the study of late lumping approaches [1] in the framework of backstepping control design for PDE-ODE cascades [23], |27].

\section{REFERENCES}

[1] J. Auriol, K. A. Morris, and F. Di Meglio, "Late-lumping backstepping control of partial differential equations," Automatica, vol. 100, pp. $247-$ 259, 2019.

[2] M. J. Balas, "Finite-dimensional controllers for linear distributed parameter systems: exponential stability using residual mode filters," Journal of Mathematical Analysis and Applications, vol. 133, no. 2, pp. 283-296, 1988.
[3] M. Barreau, F. Gouaisbaut, and A. Seuret, "Practical stability analysis of a drilling pipe under friction with a PI-controller," IEEE Transactions on Control Systems Technology, 2019.

[4] J.-M. Coron and A. Hayat, "PI controllers for 1-D nonlinear transport equation," IEEE Transactions on Automatic Control, vol. 64, no. 11, pp. 4570-4582, 2019.

[5] J.-M. Coron and E. Trélat, "Global steady-state controllability of onedimensional semilinear heat equations," SIAM Journal on Control and Optimization, vol. 43, no. 2, pp. 549-569, 2004.

[6] R. Curtain, "Finite-dimensional compensator design for parabolic distributed systems with point sensors and boundary input," IEEE Transactions on Automatic Control, vol. 27, no. 1, pp. 98-104, 1982.

[7] R. F. Curtain and H. Zwart, An introduction to infinite-dimensional linear systems theory: A State-Space Approach. Springer, 2020, vol. 71.

[8] V. Dos Santos, G. Bastin, J.-M. Coron, and B. d'Andréa Novel, "Boundary control with integral action for hyperbolic systems of conservation laws: Stability and experiments," Automatica, vol. 44, no. 5, pp. 1310$1318,2008$.

[9] C. Harkort and J. Deutscher, "Finite-dimensional observer-based control of linear distributed parameter systems using cascaded output observers," International journal of control, vol. 84, no. 1, pp. 107-122, 2011.

[10] J. P. Hespanha, Linear systems theory. Princeton university press, 2018.

[11] R. Katz and E. Fridman, "Constructive method for finite-dimensional observer-based control of 1-D parabolic PDEs," Automatica, vol. 122, p. $109285,2020$.

[12] M. Krstic and A. Smyshlyaev, Boundary control of PDEs: A course on backstepping designs. SIAM, 2008.

[13] H. Lhachemi and C. Prieur, "Finite-dimensional observer-based boundary stabilization of reaction-diffusion equations with either a Dirichlet or Neumann boundary measurement," Automatica, vol. 135, p. 109955 2022.

[14] H. Lhachemi, C. Prieur, and E. Trélat, "PI regulation of a reactiondiffusion equation with delayed boundary control," IEEE Transactions on Automatic Control, vol. 66, no. 4, pp. 1573-1587, 2020.

[15] — - "PI regulation control of a 1-D semilinear wave equation," SIAM Journal on Control and Optimization, 2021, in press.

[16] T. Meurer, Control of higher-dimensional PDEs: Flatness and backstepping designs. Springer Science \& Business Media, 2012.

[17] K. A. Morris, Controller Design for Distributed Parameter Systems. Springer, 2020.

[18] Y. Orlov, "On general properties of eigenvalues and eigenfunctions of a Sturm-Liouville operator: comments on "ISS with respect to boundary disturbances for 1-D parabolic PDEs"," IEEE Transactions on Automatic Control, vol. 62, no. 11, pp. 5970-5973, 2017.

[19] A. Pazy, Semigroups of linear operators and applications to partial differential equations. Springer Science \& Business Media, 2012, vol. 44.

[20] S. Pohjolainen, "Robust multivariable PI-controller for infinite dimensional systems," IEEE Transactions on Automatic Control, vol. 27, no. 1, pp. 17-30, 1982.

[21] D. L. Russell, "Controllability and stabilizability theory for linear partial differential equations: recent progress and open questions," SIAM Review, vol. 20, no. 4, pp. 639-739, 1978.

[22] Y. Sakawa, "Feedback stabilization of linear diffusion systems," SIAM journal on control and optimization, vol. 21, no. 5, pp. 667-676, 1983.

[23] S. Tang and C. Xie, "State and output feedback boundary control for a coupled PDE-ODE system," Systems \& Control Letters, vol. 60, no. 8, pp. 540-545, 2011

[24] A. Terrand-Jeanne, V. Andrieu, V. D. S. Martins, and C. Xu, "Adding integral action for open-loop exponentially stable semigroups and application to boundary control of PDE systems," IEEE Transactions on Automatic Control, vol. 65, no. 11, pp. 4481-4492, 2020.

[25] A. Terrand-Jeanne, V. Andrieu, M. Tayakout-Fayolle, and V. D. S. Martins, "Regulation of inhomogeneous drilling model with a PI controller," IEEE Transactions on Automatic Control, vol. 65, no. 1, pp. 58-71, 2020.

[26] N.-T. Trinh, V. Andrieu, and C.-Z. Xu, "Design of integral controllers for nonlinear systems governed by scalar hyperbolic partial differential equations," IEEE Transactions on Automatic Control, vol. 62, no. 9, pp. 4527-4536, 2017.

[27] J. Wang and M. Krstic, "Output feedback boundary control of a heat pde sandwiched between two ODEs," IEEE Transactions on Automatic Control, vol. 64, no. 11, pp. 4653-4660, 2019.

[28] C.-Z. Xu and H. Jerbi, "A robust PI-controller for infinite-dimensional systems," International Journal of Control, vol. 61, no. 1, pp. 33-45, 1995. 